Pacific Journal of Mathematics

VISUALIZING THE WORD PROBLEM, WITH AN
APPLICATION TO SIXTH GROUPS 


\title{
VISUALIZING THE WORD PROBLEM, WITH AN APPLICATION TO SIXTH GROUPS
}

\author{
C. M. Weinbaum
}

The word problem in certain groups is studied in algebraic terms with a geometric background. A relator is made to correspond to a plane complex so that generators are associated with 1-cells and defining relators are associated with 2-cells of the complex. In the case of less-than-one-sixth groups, the results obtained are essentially those found by Greendlinger.

Let $\mathscr{G}=\mathscr{F} / \mathscr{N}$ where $\mathscr{N}$ is a normal subgroup of a free group $\mathscr{F}$ with fixed free generators (understood to include inverses). Let $\mathscr{N}$ be the smallest normal subgroup containing a set $\mathscr{R}$ of cyclically reduced words (defining relators for $\mathscr{G}$ ). Nonempty words in $\mathscr{N}$ are relators for $\mathscr{G}$. Let $\mathscr{R}$ be closed under inverses and cyclic permutations. Assume each free generator appears in at least one defining relator.

In this paper we use complexes to study how relators depend upon defining relators. A complex is determined by a finite set $E$ of elements (called edges), a partition of $E$ into subsets (called boundaries), a partition of $E$ into pairs of edges, and a cyclic order for the edges in each boundary; vertices and the property of connectedness can then be defined. After a free generator is assigned to each edge (with inverse free generators assigned to paired edges), the above-mentioned cyclic orders determine words (called values) for each boundary. More precisely, some word and all its cyclic permutations are the values of a boundary.

It is shown that each relator is a value of one of the boundaries of some spherical complex (a connected complex with Euler characteristic 2) whose other boundaries have defining relators for their values. The converse is also proved: if defining relators are the values of all but one of the boundaries of a spherical complex, then a value of the remaining boundary is a relator. Thus the question of recognizing the relators in $\mathscr{G}$-the word problem in $\mathscr{G}$-can be viewed as the question of determining the words which can correspond to one boundary of a spherical complex whose other boundaries correspond to defining relators.

These results are essentially a reformulation of the first two lemmas in a paper by Van Kampen who approached the problem geometrically. The proofs given here are combinatorial in nature.

In passing from a relator to a complex, we use a system (called a

Received October 12, 1964. The work was supported by grants from the National Science Foundation, N.S.F.-GP-27 and N.S.F.-GP-1925. 
structure) which characterizes one construction of the relator from a collection of defining relators. Structures help to define certain basic relators.

The problem of recognizing relators is reduced to finding basic relators by showing that each freely reduced relator contains a subword which is a basic relator. When $W$ is a cyclically reduced basic relator, some subword of $W$ is a subword of a defining relator. The number of such subwords, contained disjointly in the cyclic word $W$, is estimated via simple calculations using a spherical complex associated with $W$. The calculations are given in $\S 8$; they were suggested by the proof of the Five Color Theorem in [1] Courant and Robbins.

This estimate is applied when $W$ is in a group $\mathscr{G}$ which is a lessthan-one-sixth group or, briefly, a sixth group. A group $\mathscr{G}$ is called a sixth group if any subword common to 2 distinct defining relators has a length which is less than one sixth of the length of both of the defining relators. As a result, $W$ is seen to contain a subword which is more than one half of a defining relator.

Thus a nonempty cyclically reduced word is a relator in a sixth group only if the word can be shortened by replacing one of its subwords $X$ by a shorter word $Y^{-1}$ where $X Y$ is a defining relator. This solves the word problem for sixth groups. Other proofs have been given by Tartakovskii and Greendlinger.

Our results are contained in the following

MAIN THEOREM. In a presented group, each freely reduced relator contains a subword which is a certain kind of relator called a basic relator.

If a cyclically reduced word $W$ is a basic relator for a sixth group, then either $W$ is a defining relator or the cyclic word $W$ contains disjointly $P_{k}$ subwords which are greater than $7-k / 6$ of $a$ defining relator $(k=2,3,4)$ and the integers $P_{k}$ satisfy $3 P_{2}+2 P_{3}+P_{4} \geqq 6$. Thus $W$ contains a subword which is more than $1 / 2$ of a defining relator.

2. Constructing relators. Let $W \cong V_{1} X V_{2}$ and $V \cong V_{1} V_{2}$ be words in $\mathscr{F}$. Here "@” stands for "identically equal to". We write $W \rightarrow V$ (delete $X$ ) and $V \rightarrow W$ (insert $X$ ). If also $V \rightarrow U$ (delete $Y$ ), then $W \rightarrow U$ (delete $X, Y$ ). This leads to a definition of $W \rightarrow W^{\prime}$ (delete $X_{1}, \cdots, X_{n}$ ) and $W^{\prime} \rightarrow W$ (insert $X_{n}, \cdots, X_{1}$ ) for $n \geqq 1$.

A word $W$ splits into one or more words $W_{1}, \cdots, W_{n}$ if the $W_{i}$ can be put in a sequence $W_{1}^{\prime}, \cdots, W_{n}^{\prime}$ so that $1 \rightarrow W\left(\right.$ insert $W_{1}^{\prime}, \cdots, W_{n}^{\prime}$ ) where 1 denotes the empty word. An $\mathscr{R}$-word of type $t$ is any word which splits into $t$ defining relators.

A product of a free generator and its inverse is a null word. If 
$W, W^{\prime}$ are words such that either $W \cong W^{\prime}$ or $W \rightarrow W^{\prime}$ (delete $N_{1}, \cdots, N_{k}$ ) where the $N_{i}$ are null words, then $W$ partially reduces to $W^{\prime}$ and $W^{\prime}$ is a partially reduced form of $W$. If, in addition, no subword of $W^{\prime}$ is a null word, then $W^{\prime}$ is the freely reduced form of $W$. A relator of type $t$ is a partially reduced $\mathscr{R}$-word of type $t$ (i.e. a partially reduced form of an $\mathscr{R}$-word of type $t$ ).

The first lemma shows that each relator can be constructed from the empty word by insertions of defining relators, possibly followed by deletions of null words.

LEMMA 2.1. Each relator is a partially reduced $\mathscr{R}$-word. In other words, each relator has at least one type.

Proof. The collection of $\mathscr{R}$-words is closed under inverses and products. If $W$ is an $\mathscr{R}$-word and $x$ is a free generator, then it must be shown that $x W x^{-1}$ is a partially reduced form of some $\mathscr{R}$-word $W^{\prime}$. Suppose $1 \rightarrow W$ (insert $R_{1}, \cdots, R_{n}$ ) where the $R_{i}$ are defining relators. Let $x$ be the first letter in a defining relator $R \cong x Y$. Put $W^{\prime} \cong$ $x W Y Y^{-1} x^{-1}$ so that $W^{\prime}$ partially reduces to $x W x^{-1}$ and $1 \rightarrow W^{\prime}$ (insert $\left.R, R_{1}, \cdots, R_{n}, R^{-1}\right)$. This completes the proof.

It can be shown if $W^{\prime \prime}$ is a cyclic permutation of a word $W$ which splits into $W_{1}, \cdots, W_{n}$, then $W^{\prime \prime}$ splits into some cyclic permutations $W_{1}^{\prime \prime}, \cdots, W_{n}^{\prime \prime}$ of $W_{1}, \cdots, W_{n}$, respectively. Hence,

REMARK 2.1. The set of $\mathscr{R}$-words of type $t$ is closed under cyclic permutations. The set of relators of type $t$ is closed under cyclic permutations.

3. Structures for relators. We need terminology for permutations of a finite set in order to define a structure. In this section, all sets are finite; 0 denotes the empty set.

Let $\theta$ be a cyclic permutation, acting on a set $E$. If $E \neq 0$, suppose $E=\left\{a_{1}, \cdots, a_{m}\right\}$ and either $m=1$ with $a_{1} \theta=a_{1}$ or $m \geqq 2$ with $a_{1} \theta=$ $a_{i+1}(1 \leqq i \leqq m-1)$ and $a_{m} \theta=a_{1}$. Then $\theta$ is represented by an array $H \cong a_{1} \cdots a_{m}$ and by the $m$ cyclic permutations of $H$. Any subword of $H$ is said to partially represent $\theta$. If $E=0$, then $\theta$ is the empty permutation, represented by the empty array 1 .

A set of words in $\mathscr{F}$ is associated with $\theta$ by assigning a free generator to each element in $E$. If $x_{i}$ is assigned to $a_{i}$, then $V \cong$ $x_{1} \cdots x_{m}$ (a word in $\mathscr{F}$ ) is called the value of $\mathrm{H}$ or a value of $\theta$. The values of $\theta$ are the cyclic permutations of $V$. If $E=0$, the empty word is the only value of $\theta$.

A cyclic permutation $\theta_{B}$ corresponds to each subset $B$ of $E$. If 
$B \neq 0$ and the elements of $B$ form a subsequence $b_{1}, \cdots, b_{k}$ of $a_{1}, \cdots$, $a_{m}$, then $\theta_{B}$ is represented by the array $b_{1} \cdots b_{k}$. If $B=0$, then $\theta_{B}$ is the empty permutation.

A permutation $\beta$, acting on a nonempty set $E$, determines a partition of $E$ into nonempty subsets $E_{1}, \cdots, E_{n}$, called $\beta$-orbits: two elements $a, b$ are in the same $\beta$-orbit if $a \beta^{i}=b$ for some integer $i$. The $\beta$-cycles are the restrictions of $\beta$ to the sets $E_{1}, \cdots, E_{n}$. The length of a $\beta$-cycle is the number of elements in the corresponding $\beta$-orbit. $\quad \beta$ is a reflection (pure reflection) if the length of each $\beta$-cycle is at most 2 (exactly 2 ).

A structure $S=(E, \beta, \rho, \theta)$ consists of a nonempty set $E$ which is acted on by a permutation $\beta$, a reflection $\rho$, and a cyclic permutation $\theta$. $S$ has carrier $E$, reduced carrier $F=\{a: a \in E, a \rho=a\}, \operatorname{map} \theta$, and reduced map $\theta_{F}$. It is required that there exist arrays $H, H_{\rho}$, representing $\theta, \theta_{F}$, respectively, such that

(I) There exist arrays $H_{1}, \cdots, H_{n}, n \geqq 1$, representing the $\beta$ cycles, such that $1 \rightarrow H$ (insert $H_{1}, \cdots, H_{n}$ ).

(II) Either $\rho$ is the identity and $H_{\rho} \cong H$ or there exist arrays $I_{1}, \cdots, I_{k}, k \geqq 1$, representing the $\rho$-cycles of length 2 , such that $H \rightarrow H_{\rho}$ (delete $I_{1}, \cdots, I_{k}$ ).

$S$ is said to be of type $n$. The members of $F$ are fixed elements; the members of $E-F$ are cancelled elements.

If $H_{\rho}$ contains a subword $I$, of length 2 , whose elements are $a, b$, then $S^{\prime}=(E, \beta, \sigma, \theta)$ is also a structure where $a \sigma=b, b \sigma=a$ and $\sigma=\rho$ except on the set $\{a, b\}$. Indeed, if $H_{\sigma}$ is defined by $H_{\rho} \rightarrow H_{\sigma}$ (delete $I$ ), then $H_{\sigma}$ represents the reduced map of $S^{\prime}$. We say that $S$ contracts to $S^{\prime}$ in one step.

$S$ is an $\mathscr{R}$-structure ( $\mathscr{N}$-structure) if a free generator is assigned to each element in $E$ in such a way that the values of the $\rho$-cycles of length 2 are null words and the values of the $\beta$-cycles are words in $\mathscr{R}$ (in $\mathscr{N}$ ). When $S$ is an $\mathscr{R}$-structure, of type $n$, with map $\theta$ and reduced map $\theta_{F}$, then the values of $\theta$ are $\mathscr{R}$-words of type $n$ and the values of $\theta_{F}$ are relators of type $n$.

THEOREM 3.1. Each relator is a value of the reduced map of some $\mathscr{R}$-structure.

Proof. Use the definition of $\mathscr{R}$-structure and Lemma 2.1.

We now turn to some more definitions concerning a structure $S=(E, \beta, \rho, \theta) . \quad S$ is called noncancelled if there exist fixed elements in $E . S$ is cancelled if $E$ contains only cancelled elements. In the latter case, $\rho$ is a pure reflection.

If $A$ is a nonempty subset of $E$, then $A$ is the carrier of a substructure $T$ whenever $A$ is closed under $\beta$ and $\rho$. In this case, $T=$ 
$\left(A, \gamma, \sigma, \theta_{A}\right)$ where $\gamma, \sigma$ are the restrictions of $\beta, \rho$, respectively, to the set $A . \quad T$ is a proper substructure if $A \neq E . \quad S$ is minimal if it has no proper substructures; $S$ is simple if it has no proper cancelled substructure.

THEOREM 3.2. Each relator is a value of the reduced map of some simple $\mathscr{R}$-structure.

Proof. Use previous theorem and next lemma.

LEMMA 3.1. Each structure has the same reduced map as some simple structure.

Proof. Consider a nonsimple structure $S=(E, \beta, \rho, \theta)$ determined by the expressions $1 \rightarrow H$ (insert $H_{1}, \cdots, H_{n}$ ) and $H \rightarrow H_{\rho}$ (delete $\left.I_{1}, \cdots, I_{k}\right)$ as in the definition of a structure. Suppose $S_{1}=\left(E_{1}, \beta_{1}, \rho_{1}, \theta_{1}\right)$ is the maximum cancelled proper substructure of $S$. Let $H^{\prime}$ denote the array that results from deleting all the elements in $E_{1}$ from $H$.

A sequence $H_{1}^{\prime}, \cdots, H_{m}^{\prime}$ remains after deleting from $H_{1}, \cdots, H_{n}$ the terms which represent the $\beta_{1}$-cycles.

A sequence $I_{1}^{\prime}, \cdots, I_{l}^{\prime}$ remains after deleting from $I_{1}, \cdots, I_{k}$ the terms which represent the $\rho_{1}$-cycles. Then the expressions $1 \rightarrow H^{\prime}$ (insert $H_{1}^{\prime}, \cdots, H_{m}^{\prime}$ ) and $H^{\prime} \rightarrow H_{\rho}$ (delete $I_{l}^{\prime}, \cdots, I_{l}^{\prime}$ ) determine a simple structure having the same reduced map as $S$.

4. Complexes. A complex $C=(E, \beta, \rho)$ consists of a finite, nonempty set $E$ which is acted on by a permutation $\beta$ and a pure reflection $\rho$. If $\alpha$ is the map $\beta$, followed by $\rho$ (i.e. $\alpha=\beta \rho$ ), then the $\alpha$-orbits, the elements in $E$, and the $\beta$-orbits are the vertices, edges, and boundaries, respectively, of $C$. Whenever a free generator is assigned to each edge, the values of the $\beta$-cycles are called the values of the boundaries of $C$.

$C$ is a disjoint union of 2 complexes $\left(E_{i}, \beta_{i}, \rho_{i}\right)$ for $i=1,2$ if $E$ is a disjoint union of $E_{1}, E_{2}$ and $\beta_{i}, \rho_{i}$ are the restrictions of $\beta, \rho$, respectively, to the set $E_{i}(i=1,2)$. If this is never the case, $C$ is said to be connected.

Since $E$ is a disjoint union of the $\rho$-orbits and each $\rho$-orbit contains exactly 2 edges, the number of edges is always even. Whenever $a$ is an edge, $a \rho$ is called the inverse of $a$. If $v, 2 e, n$ denote the numbers of vertices, edges and boundaries of $C$, then $v-e+n$ is the Euler characteristic. A spherical complex is a connected complex with Euler characteristic 2 .

Note that when $S_{1}=\left(E_{1}, \beta_{1}, \rho_{1}, \theta_{1}\right)$ is a cancelled structure, then $C_{1}=\left(E_{1}, \beta_{1}, \rho_{1}\right)$ is a complex: Furthermore, $S_{1}$ is minimal if and only if $C_{1}$ is connected. 
5. From structures to complexes. We now describe a transition from a noncancelled structure $S$ to a cancelled structure $S_{1}$; with $S_{1}$ there is associated a complex $C_{1}$.

Suppose $S=(E, \beta, \rho, \theta)$ is a noncancelled $\mathscr{R}$-structure, of type $n \geqq 1$, with $H, H_{\rho}, H_{1}, \cdots, H_{n}$ as in $\S 3$. A cancelled $\mathscr{N}$-structure $S_{1}=\left(E_{1}, \beta_{1}, \rho_{1}, \theta_{1}\right)$, of type $n+1$, is defined as follows.

Let $H_{\rho} \cong a_{1} \cdots a_{m}$. Since $S$ is noncancelled, $H_{\rho}$ is nonempty and $m \geqq 1$. Choose $m$ new elements $b_{1}, \cdots, b_{m}$; put $E_{1}=E \cup\left\{b_{1}, \cdots, b_{m}\right\}$. $\theta_{1}$ is represented by $H H_{n+1}$ where $H_{n+1} \cong b_{m} \cdots b_{1}$. Then $H_{p} H_{n+1} \rightarrow 1$ (delete $J_{1}, \cdots, J_{m}$ ) where $J_{i} \cong a_{i} b_{i}(1 \leqq i \leqq m)$. The $\beta_{1}$-cycles are represented by $H_{1}, \cdots, H_{n}, H_{n+1}$. The $\beta_{1}$-cycle represented by $H_{n+1}$ is called the distinguished $\beta_{1}$-cycle of $S_{1}$.

If $\rho$ is the identity, then the $\rho_{1}$-cycles are represented by $J_{1}, \cdots, J_{m}$. If $\rho$ is not the identity, then we have $H \rightarrow H_{\rho}$ (delete $I_{1}, \cdots, I_{k}$ ) where the $I_{i}$ represent the $\rho$-cycles of length 2 . In this case, $H H_{n+1} \rightarrow 1$ (delete $I_{1}, \cdots, I_{k}, J_{1}, \cdots, J_{m}$ ) and the $I_{i}, J_{i}$ represent the $\rho_{1}$-cycles.

A free generator is assigned to each $b_{i}$ so that the values of $H_{\rho}$ and $H_{n+1}$ are inverse words. This insures that the values of the $J_{i}$ are null words and the value of $H_{n+1}$ is a relator. The $\mathfrak{N}^{\text {-structure }}$ $S_{1}$ is now complete and $C_{1}=\left(E_{1}, \beta_{1}, \rho_{1}\right)$.

With reference to the construction of $S_{1}$, we have:

REMARK 5.1. If $a b$ is a subword, of length 2, of some cyclic permutation of $H_{\rho}$ and if $a \rho_{1}=c, b \rho_{1}=d$, then $d c$ is a subword of some cyclic permutation of $H_{n+1}$. In other words, if $a, b$ are distinct fixed elements of $S$ and $a \theta_{F}=b$ where $\theta_{F}$ is the reduced map of $S$, then $b \rho_{1} \beta_{1}=a \rho_{1}$.

Lemma 5.1. If $S$ is simple or minimal, then $S_{1}$ is minimal.

Proof. Since minimal implies simple for structures, we assume $S$ is simple. Suppose a nonempty proper subset $A_{1}$ (of $E_{1}$ ) is closed under $\beta_{1}$ and $\rho_{1}$. Then $A_{2}=E_{1}-A_{1}$ also has this property; $A_{1}, A_{2}$ are carriers of substructures of $S_{1}$. Thus all the elements $b_{i}$ are in the same $A_{j}$, say in $A_{2}$. Therefore all the elements in $A_{1}$ are cancelled elements in $S$. But then $A_{1}$ is the carrier of a proper cancelled substructure of $S$, contary to the assumption that $S$ is simple.

THEOREM 5.1. For each relator $W$ there is a cancelled, minimal $\mathcal{N}$-structure $S_{1}=\left(E_{1}, \beta_{1}, \rho_{1}, \theta_{1}\right)$, of type $t \geqq 2$ and a connected complex $C_{1}=\left(E_{1}, \beta_{1}, \rho_{1}\right)$ such that the $\beta_{1}$-cycles can be represented by $t$ arrays whose values are $W^{-1}$ and $t-1$ defining relators. 
Proof. By Theorem 3.2 there is a simple $\mathscr{R}$-structure $S$, of type $n \geqq 1$, where $W$ is one of the values of the reduced map of $S$. Earlier we constructed a cancelled $\mathscr{N}$-structure $S_{1}=\left(E_{1}, \beta_{1}, \rho_{1}, \theta_{1}\right)$, of type $n+1$, whose $\beta_{1}$-cycles satisfy the desired condition. By Lemma $5.1 S_{1}$ is minimal; hence, $C_{1}=\left(E_{1}, \beta_{1}, \rho_{1}\right)$ is connected.

6. Spherical complexes. The relationship between relators and spherical complexes is given in Theorem 6.2 and in Theorem 6.4. Their proofs depend on Theorem 6.1 and Theorem 6.3, which are converses. Three preliminary lemmas are needed.

LEMMA 6.1. Let $H_{1}, \cdots, H_{n}$ be arrays with disjoint sets of elements satisfying $1 \rightarrow H\left(\right.$ insert $H_{1}, \cdots, H_{n}$ ) where $H$ is an array and $n \geqq 2$. Suppose $H$ has a subword $I$, of length 2, whose letters $a, b$ are in $H_{i}, H_{j}$, respectively, for $i<j$. Then $1 \rightarrow H$ (insert $H_{1}, \cdots, H_{i-1}, K$, $\left.H_{i+1}, \cdots, H_{j-1}, H_{j+1}, \cdots, H_{n}\right)$ for some array $K$, having subword $I$, such that $1 \rightarrow K\left(\right.$ insert $\left.H_{i}, H_{j}\right)$.

Proof. Let $H^{\prime}$ be the array such that $1 \rightarrow H^{\prime}$ (insert $H_{1}, \cdots, H_{i}$, $\cdots, H_{j}$ ) and $H^{\prime} \rightarrow H$ (insert $H_{j+1}, \cdots, H_{n}$ ). Then $I$ is a subword of $H^{\prime}$. We also have $1 \rightarrow H^{\prime}$ (insert $H_{1}, \cdots, H_{i-1}, H_{i}, H_{j}, H_{i+1}, \cdots, H_{j-1}$ ). Let $H_{i} \cong A_{1} a A_{2}$ and $H_{j}=B_{2} b B_{1}$.

If $I \cong a b$, then $B_{2}$ is the empty array and we put $K \cong A_{1} a b B_{1} A_{2}$. If $I \cong b a$, then $B_{1}$ is the empty array and we put $K \cong A_{1} B_{2} b a A_{2}$.

Lemma 6.2. Let the array $a b c_{1} \cdots c_{r}(r \geqq 1)$ represent $a \beta$-cycle $\mu$ corresponding to a $\beta$-orbit $B$ of a connected complex $C=(E, \beta, \rho)$. Assume $a \rho=b$. Then $C$ has the same Euler characteristic as some connected complex $C^{\prime}=\left(E^{\prime}, \beta^{\prime}, \rho^{\prime}\right)$ having 2 fewer edges than $C$.

Proof. Put $B^{\prime}=\left\{c_{1}, \cdots, c_{r}\right\}$ and $E^{\prime}=E-\{a, b\}$. Let $\mu^{\prime}$ be the cyclic permutation represented by the array $c_{1} \cdots c_{r}$. Define $\rho^{\prime}$ to be the restriction of $\rho$ to the set $E^{\prime}$. Define $\beta^{\prime}$ by putting $\beta^{\prime}=\mu^{\prime}$ on $B^{\prime}$ and $\beta^{\prime}=\beta$ on $E^{\prime}-B^{\prime}$. The connectedness of $C^{\prime}$ follows from the connectedness of $C$. Thus, it suffices to show that $C$ has one more vertex than $C^{\prime}$.

Since $a \beta \rho=b \rho=a,\{a\}$ is a vertex of $C . \quad c_{r}$ is the only edge in $E^{\prime}$ having different images under $\beta \rho$ and $\beta^{\prime} \rho^{\prime}$. In fact, $c_{r} \beta \rho=a \rho=b$ and $c_{r} \beta^{\prime} \rho^{\prime}=c_{1} \rho^{\prime}=c_{1} \rho$. Furthermore $b \neq c_{1} \rho$ since $a \neq c_{1}$ and $a \rho=b$.

Let $d=c_{1} \rho, \alpha=\beta \rho, \alpha^{\prime}=\beta^{\prime} \rho^{\prime}$. There is an $\alpha$-orbit $V$ whose $\alpha$ cycle is represented by an array of the form $c_{r} b d D$ and $V$ is a disjoint union of $\{b\}$ and an $\alpha^{\prime}$-orbit $V^{\prime}$ whose $\alpha^{\prime}$-cycle is represented by $c_{r} d D$. Thus $C, C^{\prime}$ have the same vertices, except that $\{a\}$ and $V$ in $C$ are replaced by $V^{\prime}$ in $C^{\prime}$. This completes the proof. 
LEMMA 6.3. Let $C=(E, \beta, \rho)$ be a connected complex with $n \geqq 2$ boundaries. Let $A=\left\{a_{1}, \cdots, a_{r}\right\}, B=\left\{b_{1}, \cdots, b_{s}\right\}$ be $\beta$-orbits whose $\beta$-cycles $\mu, \nu$ are represented by arrays $a_{1} \cdots a_{r}$ and $b_{1} \cdots b_{s}$, respectively. Assume $b_{1} \rho=a_{r}$. Then $C$ has the same Euler characteristic as some connected complex $C^{\prime}=\left(E, \beta^{\prime}, \rho\right)$ having $n-1$ boundaries.

Proof. Let $\mu^{\prime}$ be the cyclic permutation represented by the array $a_{1} \cdots a_{r} b_{1} \cdots b_{s}$. Define $\beta^{\prime}$ by putting $\beta^{\prime}=\mu^{\prime}$ on the set $A \cup B$ and $\beta^{\prime}=\beta$ otherwise. Then $C$ has one more boundary than $C^{\prime}$ since 2 $\beta$-orbits $A, B$ are replaced by one $\beta^{\prime}$-orbit $A \cup B$. We must show that $C^{\prime}$ has one more vertex than $C$ (i.e. that $\beta^{\prime} \rho$ has one more orbit than $\beta \rho$ ).

Only $b_{s}=b_{1} \beta^{-1}$ and $a_{r}$ have different images under $\beta \rho$ than under $\beta^{\prime} \rho$. In fact $b_{s} \beta \rho=b_{1} \rho=a_{r}$ and $b_{s} \beta^{\prime} \rho=a_{1} \rho ; a_{r} \beta \rho=a_{1} \rho$ and $a_{r} \beta^{\prime} \rho=$ $b_{1} \rho=a_{r}$. Furthermore $a_{1} \rho \neq a_{r}$ since $a_{1} \neq b_{1}$ and $b_{1} \rho=a_{r}$.

Let $c=a_{1} \rho, \alpha=\beta \rho$, and $\alpha^{\prime}=\beta^{\prime} \rho$. There is an $\alpha$-orbit $V$ whose $\beta$-cycle is represented by an array of the form $b_{s} a_{r} c D$ and $V$ is a disjoint union of $2 \alpha^{\prime}$-orbits $V^{\prime}, V^{\prime \prime}$ whose $\alpha^{\prime}$-cycles are represented by the arrays $a_{r}$ and $b_{s} c D$. Thus $C, C^{\prime}$ have the same vertices, except that $V$ is replaced by $V^{\prime}$ and $V^{\prime \prime}$. Therefore $C^{\prime}$ has one more vertex than $C$.

The connectedness of $C^{\prime}$ follows from the connectedness of $C$.

THEOREM 6.1. Let $S=(E, \beta, \rho, \theta)$ be a minimal, cancelled structure of type $n \geqq 1$. Then $C=(E, \beta, \rho)$ is a spherical complex.

Proof. Use induction on the number $2 e$ of edges of $C$. Suppose $2 e=2$. Then $E=\{a, b\}$ and $a \rho=b, b \rho=a$; hence $C$ is connected. If the $\beta$-orbits are $\{a\}$ and $\{b\}$ so that $n=2$, then $a \beta \rho=a \rho=b$, $b \beta \rho=b \rho=a$ and $\{a, b\}$ is the only vertex. Thus $v-e+n=1-1+2=2$. If $\{a, b\}$ is the only $\beta$-orbit so that $n=1$, then $a \beta \rho=b \rho=a, b \beta \rho=$ $a \rho=b$ and $\{a\},\{b\}$ are the only vertices. Thus, $v-e+n=2-1+1=2$.

Now assume that $2 e \geqq 4$ and that the theorem holds for complexes with fewer than $2 e$ edges. Let $H, H_{1}, \cdots, H_{n}$ represent $\theta$ and the $\beta$-cycles and let $I_{1} \cong a b, I_{2}, \cdots, I_{e}$ represent the $\rho$-cycles. Assume that

$$
\begin{aligned}
& 1 \rightarrow H \text { (insert } H_{1}, \cdots, H_{n} \text { ) } \\
& H \rightarrow 1 \text { (delete } I_{1}, \cdots, I_{e} \text { ). }
\end{aligned}
$$

Suppose $a$ is in $H_{i}, b$ is in $H_{j}$.

Case 1. $(i=j)$ Then $I_{1}$ is a subword of $H_{i}$. Let $\beta_{1}$ be the $\beta$-cycle represented by $H_{i}$. It cannot happen that $H_{i} \cong I_{1}$ since then $C_{1}=\left(E_{1}, \beta_{1}, \rho_{1}\right)$ is a subcomplex of $C$ where $E_{1}=\{a, b\}$ and $I_{1}$ represents the only $\rho_{1}$-cycle. Also $S$ is minimal so $C$ is connected; hence $C=C_{1}$. This is contrary to $2 e \geqq 4$. Therefore, some cyclic permutation of $H_{i}$ is of the form $a b c_{1} \cdots c_{r}(r \geqq 1)$. 
A minimal, cancelled structure $S^{\prime}=\left(E^{\prime}, \beta^{\prime}, \rho^{\prime}, \theta^{\prime}\right)$ is determined as follows. Let $H^{\prime}, H_{1}, \cdots, H_{i-1}, H_{i}^{\prime}, H_{i+1}, \cdots, H_{n}$ represent $\theta^{\prime}$ and the $\beta^{\prime}$-cycles, and $I_{2}, \cdots, I_{e}$ represent the $\rho^{\prime}$-cycles, where

$$
\begin{aligned}
H & \rightarrow H^{\prime} \quad\left(\text { delete } I_{1}\right) \\
H_{i} & \rightarrow H_{i}^{\prime}\left(\text { delete } I_{1}\right) \\
1 & \rightarrow H^{\prime} \quad\left(\text { insert } H_{1}, \cdots, H_{i-1}, H_{i}^{\prime}, H_{i+1}, \cdots, H_{n}\right) \\
H^{\prime} & \rightarrow 1 \quad\left(\text { delete } I_{2}, \cdots, I_{e}\right) .
\end{aligned}
$$

The complexes $C^{\prime}=\left(E^{\prime}, \beta^{\prime}, \rho^{\prime}\right)$ and $C$ have the same Euler characteristic and $C^{\prime}$ is connected by Lemma 6.2.

Case 2. $(i \neq j)$ Suppose $i<j$ (Treatment of $j<i$ is similar.) A minimal, cancelled structure $S^{\prime}=\left(E, \beta^{\prime}, \rho, \theta\right)$ is determined as follows. Let $H_{1}, \cdots, H_{i-1}, K, H_{i+1}, \cdots, H_{j-1}, H_{j+1}, \cdots, H_{n}$ ) represent the $\beta^{\prime}$-cycles where $K$ has the subword $I_{1}$ and

$$
\begin{aligned}
& 1 \rightarrow H\left(\text { insert } H_{1}, \cdots, H_{i-1}, K, H_{i+1}, \cdots, H_{j-1}, H_{j+1}, \cdots, H_{n}\right. \text { ) } \\
& 1 \rightarrow K \text { (insert } H_{i}, H_{j} \text { ). }
\end{aligned}
$$

This is possible by Lemma 6.3.

The complexes $C^{\prime}=\left(E, \beta^{\prime}, \rho\right)$ and $C$ have the same Euler characteristic and $C^{\prime}$ is connected (by Lemma 6.1). In fact, some cyclic permutation of $K, H_{i}$, and $H_{j}$ are of the forms $a_{1} \cdots a_{r} b_{1} \cdots b_{s}, a_{1} \cdots a_{r}$, and $b_{1} \cdots b_{s}$, respectively, where $I_{1} \cong a_{r} b_{1}$. Now $S^{\prime}$ and $C^{\prime}$ can be treated as in Case 1 , since $I_{1}$ is a subword of $K$.

Thus, in either one or two steps, we can always find a new minimal, cancelled structure whase associated connected complex has $2(e-1)$ edges such that the original and new complexes have the same Euler characteristic. By the induction assumption, the new complex has Euler characteristic 2; hence, so does the original complex. This completes the proof.

THEOREM 6.2. For each relator $W$ there is some spherical complex $C$ with $n \geqq 2$ boundaries such that a free generator is assigned to each edge (with inverse free generators assigned to inverse edges), $W^{-1}$ is a value of one of the boundaries, and defining relators are the values of the remaining boundaries.

Proof. Use Theorem 5.1 and Theorem 6.1.

THEOREM 6.3. Let $C=(E, \beta, \rho)$ be a spherical complex with $n \geqq 1$ boundaries. Then there exists some minimal, cancelled structure $S=(E, \beta, \rho, \theta)$. 
Proof. Use induction on $n$. We first prove the case $n=1$. Here $\beta$ itself is the only $\beta$-cycle. This case will be proved by induction on the number $2 e$ of edges. When $2 e=2$, we have $\beta=\rho$ and we take $\theta=\beta$.

Now assume $n=1,2 e \geqq 4$ and the theorem holds for complexes having one boundary and fewer than $2 e$ edges. There must be a vertex containing just one edge since, if not, we have $2 e \geqq 2 v$ and $v-e+1=2$ (where $v$ is the number of vertices). But this implies $e \geqq v$ and $v=1+e$ which is impossible. If $\{a\}$ is a vertex, let $b=a \rho$. Then $a \beta=b$ since $a \beta \rho=a$. Thus $\beta$ is represented by some array $H \cong I_{1} H^{\prime}$ where $I_{1} \cong a b$.

A connected complex $C^{\prime}=\left(E^{\prime}, \beta^{\prime}, \rho^{\prime}\right)$ with $2 e-2$ edges and 1 boundary is defined by $E^{\prime}=E-\{a b\}$ if we take $\rho^{\prime}$ to be the restriction of $\rho$ to $E^{\prime}$ and put $\beta^{\prime}=\beta_{A}$ with $A=E^{\prime}$. Now apply the induction assumption to $C^{\prime}$. There exists a minimal, cancelled structure $S^{\prime}=$ $\left(E^{\prime}, \beta^{\prime}, \rho^{\prime}, \theta^{\prime}\right)$. There exist an array $X$ representing $\theta^{\prime}=\beta^{\prime}$ and arrays $I_{2}^{\prime}, \cdots, I_{e}^{\prime}$ representing the $\rho^{\prime}$-cycles such that $X \rightarrow 1$ (delete $I_{2}^{\prime}, \cdots, I_{e}^{\prime}$ ). But since $H^{\prime}$ is a cyclic permutation of $X$, there exist arrays $I_{2}, \cdots, I_{e}$ representing the $\rho^{\prime}$-cycles such that $H^{\prime} \rightarrow 1$ (delete $I_{2}, \cdots, I_{e}$ ).

Since $H \rightarrow H^{\prime}$ (delete $I_{1}$ ), we have that $\theta=\beta$ is represented by an array $H$ satisfying $H \rightarrow 1$ (delete $\left.I_{1}, I_{2}, \cdots, I_{e}\right)$. Thus $S=(E, \beta, \rho, \theta)$ is a cancelled structure which is minimal since $C$ is connected.

Now suppose $n \geqq 2$. Assume that the theorem holds for complexes having fewer than $n$ boundaries. We need only consider the case that there exist two edges $a, b$, in different boundaries, such that $a \rho=b$. For if an edge and its image under $\rho$ are always in the same boundary, then one boundary $E_{1}$ consists of the edges in some subcomplex which must be the whole complex $C$, by the connectedness of $C$. But then $n=1$.

Thus, we can choose two $\beta$-cycles $\mu, \nu$ represented by arrays $a_{1} \cdots a_{r}$ and $b_{1} \cdots b_{s}$, respectively, such that $a_{r} \rho=b_{1}$. Form a connected complex $C^{\prime}=\left(E, \beta^{\prime}, \rho\right)$, having $n-1$ boundaries, as in Lemma 6.3. The induction assumption implies that there is a minimal, cancelled structure $S^{\prime}=\left(E, \beta^{\prime}, \rho, \theta\right)$. Here one of the $\beta^{\prime}$-cycles $\mu^{\prime}$ is represented by the array $a_{1} \cdots a_{r} b_{1} \cdots b_{s}$. There exist arrays $H, H_{1}$, $\cdots, H_{n-1}$ representing $\theta$ and the $n-1 \beta^{\prime}$-cycles such that $1 \rightarrow H$ (insert $\left.H_{1}, \cdots, H_{n-1}\right)$. Then $a_{1} \cdots a_{r} b_{1} \cdots b_{s}$ is a cyclic permutation of $H_{i}$, for some $i$. Thus $1 \rightarrow H_{i}$ (insert $A, B$ ) or $1 \rightarrow H_{i}$ (insert $B, A$ ) for some arrays $A, B$ which are cyclic permutations of $a_{1} \cdots a_{r}$ and $b_{1} \cdots b_{s}$, respectively. In either case, $H$ splits into $H_{1}, \cdots, H_{i-1}, A, B, H_{i+1}$, $\cdots, H_{n-1}$ which represent the $\beta$-cycles. Thus $S=(E, \beta, \rho, \theta)$ is a cancelled structure which is minimal since $C$ is connected.

ThEOREM 6.4. Let $C=(E, \beta, \rho)$ be a spherical complex with $n \geqq 2$ 
boundaries such that a free generator is assigned to each edge (with inverse free generators assigned to inverse edges). If all but one of the boundaries have values which are defining relators, then each value of the remaining boundary is a relator.

Proof. A minimal, cancelled structure $S=(E, \beta, \rho, \theta)$ exists by Theorem 6.3. Suppose an array $H$ represents $\theta$. Since $H$ splits into arrays representing the $\rho$-cycles, $W$ splits into null words so that $W$ is a relator. Since $H$ splits into arrays representing the $\beta$-cycles, $W$ splits into $n-1$ defining relators and a word $K$ (a value of the "remaining" $\beta$-cycle). Since $W$ is a relator, $K$ must be a relator.

7. Sides of nontrivial complexes. In this section each complex $C=(E, \beta, \rho)$ is nontrivial (i.e. has $n \geqq 3$ boundaries). When $C$ is also spherical, we show that each $\beta$-cycle can be represented by an array which is broken up into a product $X_{1} \cdots X_{t}(t \geqq 1)$ where each $X_{i}$ has certain properties. The $X_{i}$ will be called sides. In order to define sides, we classify the edges of $C$. Let $a$ be an edge.

If either $a \rho \beta=a$ or $a \rho \beta \rho \beta \neq a$, then $a$ is initial. If either $a \beta \rho=a$ or $a \beta \rho \beta \rho \neq a$, then $a$ is final. Thus, if $a$ is initial, final, or neither, then $a \rho$ is final, initial, or neither, respectively. Also, if $a$ is initial, then $a \beta^{-1}$ is final; if $a$ is final, then $a \beta$ is initial.

An array $X \cong a_{1} \cdots a_{r}(r \geqq 1)$, which partially represents a $\beta$-cycle, is a side if $a_{1}$ is the only initial edge in $X$ and $a_{r}$ is the only final edge in $X$. If $X \cong a_{1} \cdots a_{r}$ is a side, then the array $Y \cong b_{r} \cdots b_{1}$, where $a_{i} \rho=b_{i}(1 \leqq i \leqq r)$, is called the inverse of $X$.

LEMmA 7.1. If $X \cong a_{1} \cdots a_{r}$ is a side, so is its inverse $Y \cong b_{r} \cdots b_{1}$.

Proof. It suffices to check that $Y$ partially represents a $\beta$-cycle when $r \geqq 2$. i.e. $b_{i+1} \beta=b_{i}$ for $1 \leqq i \leqq r-1$. Indeed, $b_{i+1} \beta=a_{i+1} \rho \beta=$ $a_{i} \beta \rho \beta=b_{i} \rho \beta \rho \beta=b_{i}$. The last equality holds since $b_{i}$ is not initial for $1 \leqq i \leqq r-1$.

Lemma 7.2. Let $C=(E, \beta, \rho)$ be a connected complex with $n \geqq 3$ boundaries. Then each boundary contains at least one initial edge and at least one final edge (possibly the same edge).

Proof. Suppose the array $A \cong a_{1} \cdots a_{r}, r \geqq 1$, represents a $\beta$ cycle so that $\left\{a_{1}, \cdots, a_{r}\right\}$ is a boundary. Let $B \cong b_{r} \cdots b_{1}$ be the inverse of $A$. Suppose all the $a_{i}$ are not final. Then all the $b_{i}$ are not initial.

When $r \geqq 2, b_{i+1} \beta=b_{i}$ for $1 \leqq i \leqq r-1$ as in the proof of the previous lemma. $b_{1} \beta=a_{1} \rho \beta=a_{r} \beta \rho \beta=b_{r} \rho \beta \rho \beta=b_{r}$. When $r=1$, 
$a_{1} \beta=a_{1}$ and $b_{1} \beta=b_{1} \rho \beta \rho \beta=b_{1}$. In either case, $E_{1}=\left\{a_{1}, \cdots, a_{r}, b_{1}, \cdots, b_{r}\right\}$ is closed under $\beta$ and $\rho$. Hence $C_{1}=\left(E_{1}, \beta_{1}, \rho_{1}\right)$ is a subcomplex where $\beta_{1}, \rho_{1}$ are the restrictions of $\beta, \rho$ to $E_{1} . \quad C_{1}$ must be the whole complex by the connectedness of $C$. But $C_{1}$ has just two boundaries: $\left\{a_{1}, \cdots\right.$, $\left.a_{r}\right\}$ and $\left\{b_{1}, \cdots, b_{r}\right\}$. This contradicts $n \geqq 3$. Thus some $a_{i}$ is final and then $a_{i} \beta$ is initial.

LEMMA 7.3. Let $C=(E, \beta, \rho)$ be a connected complex with $n \geqq 3$ boundaries. Then each $\beta$-cycle can be represented by a product $X_{1} \cdots X_{t}(t \geqq 1)$ where each $X_{i}$ is a side. This representation is unique to within a cyclic permutation of these sides.

Proof. Let $\mu$ be a $\beta$-cycle. Choose an array $M$, representing $\mu$, so that the first letter of $M$ is an initial edge. (Then the last letter of $M$ is a final edge.) Therefore $M \cong X_{1} \cdots X_{t}, t \geqq 1$, where an edge in $M$ is initial (final) if and only if it is the first (last) letter in some $X_{i}$. The essential uniqueness of this representation follows from the fact that each edge can be placed uniquely in one of four classes: initial but not final, neither initial nor final, final but not initial and both initial and final. This completes the proof.

Vertices containing exactly 2 edges are called nonessential; all other vertices are essential. If the inverse arrays $X \cong a_{1} \cdots a_{r}$ and $Y \cong b_{r} \cdots b_{1}(r \geqq 2)$ are sides, then $\left\{a_{i}, b_{i+1}\right\}$ are nonessential vertices for $1 \leqq i \leqq r-1$ since $a_{i} \beta \rho=a_{i+1} \rho=b_{i+1}$ and $b_{i+1} \beta \rho=b_{i} \rho=a_{i}$. The next lemma shows that all nonessential vertices arise in this way.

LEMMA 7.4. If $\left\{a_{1}, b_{2}\right\}$ is a nonessential vertex of a complex $C=(E, \beta, \rho)$ and if $a_{2}=a_{1} \beta, b_{1}=b_{2} \beta$, then $a_{1} a_{2}$ and $b_{2} b_{1}$ are subwords of sides.

Proof. $a_{2} \rho=a_{1} \beta \rho=b_{2} ; b_{1} \rho=b_{2} \beta \rho=a_{1}$. We must show that $a_{1}$ is not final and $a_{2}$ is not initial. Indeed, $a_{1} \beta \rho \neq a_{1}$ since $b_{2} \neq a_{1}$; $a_{1} \beta \rho \beta \rho=a_{2} \rho \beta \rho=b_{2} \beta \rho=a_{1}$. Also, $a_{2} \rho \beta \neq a_{2}$ since $a_{2} \rho \beta=b_{2} \beta=b_{1}$ and $b_{1} \rho=a_{1} \neq b_{2}=a_{2} \rho$. Similarly, $b_{2}$ is not final and $b_{1}$ is not initial. This completes the proof.

The relationships between essential vertices, final edges, and sides can now be given.

Lemma 7.5. Let $C=(E, \beta, \rho)$ be a nontrival complex. An edge is in an essential vertex if and only if the edge is final. An edge is final if and only if it is the last letter in some side.

Proof. Let $a$ be an edge. Suppose $a$ is in an essential vertex $V$. If $V=\{a\}$, then $a \beta \rho=a$ and $a$ is final. If $V$ contains at least 3 
edges, then $a, b=a \beta \rho$ and $c=b \beta \rho$ are distinct edges. Thus, $a \beta \rho \beta \rho=$ $c \neq a$; hence, $a$ is final.

Now suppose $a$ is final. If $a \beta \rho=a$, then $\{a\}$ is a vertex. If $a \beta \rho \beta \rho \neq a$, then $b=a \beta \rho \neq a$ and $c=b \beta \rho \neq a$. Also $a \neq b$ and the fact that $\beta \rho$ is a one-to-one map imply that $b=a \beta \rho \neq b \beta \rho=c$. Therefore there is an essential vertex containing $a, b, c$ among its edges. The second statement of Lemma 7.5 follows from the proof of Lemma 7.3.

THEOREM 7.1. Let $C=(E, \beta, \rho)$ be the connected complex associated with a cancelled, minimal $\mathscr{N}$-structure $S=(E, \beta, \rho, \theta)$, of type $n \geqq 3$. Assume that the values of the $\beta$-cycles are cyclically reduced words. Let $2 s, w$ denote the number of sides and the number of essential vertices of $C$. Then there is no vertex containing just one edge, $2 s \geqq 3 w$, and $w-s+n=2$.

Proof. If $\{a\}$ were a vertex, then $a \beta \rho=a$; hence $a \beta=a \rho$. Let $b=a \beta$. Then $a b$ partially represents some $\beta$-cycle $\mu$. Since $a \rho=b$, the value of $a b$ is a null word which is a subword of a value of $\mu$. This contradicts the assumption that the values of the $\beta$-cycles are cyclically reduced words. Hence, there is no vertex $\{a\}$.

Therefore each essential vertex contains at least 3 edges. Using Lemma 7.5 and the resulting fact that there is a one-to-one correspondence between final edges and sides, we get $2 s \geqq 3 w$.

We know that $v-e+n=2$ where $v, 2 e$ are the numbers of vertices and edges of $C$. We show that $v-e=w-s$ by letting each pair of inverse sides (of length $m \geqq 2$ ) replace $2 m$ edges and $m-1$ nonessential vertices. In fact, if $X \cong a_{1} \cdots a_{m}, Y \cong b_{m} \cdots b_{1}$ are inverse sides $(m \geqq 2)$, then the letters in $X, Y$ are the discarded edges and $\left\{a_{i}, b_{i+1}\right\}$ for $1 \leqq i \leqq m-1$ are the discarded vertices. Thus each step reduces both $v$ and $e$ by $m-1$. Lemma 7.4 assures us that each nonessential vertex (if any) will be discarded in this process. After a finite number of steps, we have discarded all edges which are not sides and all nonessential vertices. Thus $v-e=w-s$ and $w-s+n=2$.

8. Calculations. Let $S=(E, \beta, \rho, \theta)$ be a noncancelled, minimal $\mathscr{R}$-structure, of type $n \geqq 2$, with reduced map $\theta_{F}$. Assume that the values of $\theta_{F}$ are cyclically reduced words. Let $S_{1}=\left(E_{1}, \beta_{1}, \rho_{1}, \theta_{1}\right)$ be a cancelled, minimal $\mathscr{N}$-structure, of type $n+1$, associated with $S$. (Thus the values of the $\beta_{1}$-cycles are cyclically reduced words.) Suppose that the distinguished $\beta_{1}$-cycle has $m$ sides in the complex $C_{1}=\left(E_{1}, \beta_{1}, \rho_{1}\right)$.

Consider a side $X$ of a nondistinguished $\beta_{1}$-cycle of $C_{1}$. $X$ will be called a fixed side whenever the inverse of $X$ is a side of the distinguished $\beta_{1}$-cycle. In such a case, the letters in $X$ are all fixed elements in $E$. 
Let $B_{k}^{i}$ denote the number of nondistinguished boundaries having $k$ sides, $i$ sides of which are fixed; put $B_{k}=\sum_{i} B_{k}^{i}$. Then we have

$$
\begin{aligned}
& n=\sum_{k=1}^{\infty} B_{k} ; \quad 2 s=m+\sum_{k=1}^{\infty} k B_{k} \\
& m=\sum_{k=1}^{\infty} \sum_{1 \leq i \leq k} i B_{k}^{i} .
\end{aligned}
$$

From Theorem 7.1 applied to $C_{1}$ we get $6 w-6 s+6(n+1)=12$ and $4 s \geqq 6 w$. Therefore

$$
6 n-2 s \geqq 6 .
$$

From (1) and (2) we get

$$
\sum_{k=1}^{5}(6-k) B_{k} \geqq m+6+\sum_{k=7}^{\infty}(k-6) B_{k}
$$

and

$$
\sum_{k=1}^{5}(6-k) B_{k} \geqq m+6 .
$$

Now expand the left hand side of (3):

$$
\begin{aligned}
\sum_{k=1}^{5}(6-k) B_{k}=\sum_{k=1}^{4}(5-k) B_{k}^{1} & +\sum_{k=1}^{5} B_{k}^{1}+\sum_{k=1}^{5}(6-k) B_{k}^{0} \\
& +\sum_{k=2}^{5} \sum_{i=2}^{k}(6-k) B_{k}^{i} .
\end{aligned}
$$

Further,

$$
\sum_{k=2}^{5} \sum_{i=2}^{k}(6-k) B_{k}^{i} \leqq 2 B_{2}^{2}+B_{3}^{2}+\sum_{k=2}^{5} \sum_{i=2}^{k} i B_{k}^{i} .
$$

This can be seen as follows:

When $(i, k)$ is neither $(2,2)$ nor $(2,3)$, we have $(6-k) \leqq i$.

When $i=k=2,(6-k) B_{k}^{i}=2 B_{2}^{2}+i B_{k}^{i}$.

When $i=2, k=3,(6-k) B_{k}^{i}=B_{3}^{2}+i B_{k}^{i}$.

Now use (3), (4), and (5) to get:

$$
\begin{aligned}
\sum_{k=1}^{4}(5-k) B_{k}^{1} & +\sum_{k=1}^{5}(6-k) B_{k}^{0}+\sum_{k=1}^{5} B_{k}^{1}+2 B_{2}^{2}+B_{3}^{2} \\
& +\sum_{k=2}^{5} \sum_{i=2}^{k} i B_{k}^{i} \geqq m+6 .
\end{aligned}
$$

But

$$
m \geqq \sum_{k=1}^{5} B_{k}^{1}+\sum_{k=2}^{5} \sum_{i=2}^{k} i B_{k}^{i}
$$

Therefore, 


$$
\sum_{k=1}^{4}(5-k) B_{k}^{1}+\sum_{k=1}^{5}(6-k) B_{k}^{0}+2 B_{2}^{2}+B_{3}^{2} \geqq 6
$$

9. Minimal relators. A minimal relator of type $n$ is a value of the reduced map of a minimal $\mathscr{R}$-structure of type $n$ (i.e. a minimal structure, of type $n$, which is also an $\mathscr{R}$-structure). Similarly a nonminimal relator corresponds to a nonminimal $\mathscr{R}$-structure.

We aim to show that each relator splits into minimal relators. We prove this by showing that an analogous situation holds for the reduced map of a structure $S$ and the reduced maps $\theta_{1}, \cdots, \theta_{r}$ of the minimal substructures of $S$. This requires the following.

Definition. Let $\theta, \theta_{1}, \cdots, \theta_{r}$ be cyclic permutations acting on sets $E, E_{1}, \cdots, E_{r}$, respectively, such that $E=E_{1} \cup \cdots \cup E_{r}$ is a disjoint union $(r \geqq 1)$. $\theta$ splits into $\theta_{1}, \cdots, \theta_{r}$ if the $\theta_{i}$ can be put in a sequence $\theta_{1}^{\prime}, \cdots, \theta_{r}^{\prime}$ and if arrays $H, H_{1}, \cdots, H_{r}$, representing $\theta, \theta_{1}^{\prime}, \cdots, \theta_{r}^{\prime}$, respectively, can be chosen so that $1 \rightarrow H$ (insert $H_{1}, \cdots, H_{r}$ ).

THEOREM 9.1. The reduced map of any structure $S$ splits into the reduced maps of the minimal substructures of $S$.

The proof of Theorem 9.1 requires a lemma.

Lemma 9.1. Suppose the structure $S=(E, \beta, \rho, \theta)$ contracts to the structure $S^{\prime}=(E, \beta, \sigma, \theta)$ in one step. If $S$ satisfies Theorem 9.1, so does $S^{\prime}$.

Proof. By assumption there exist arrays $H_{\rho}, H_{\sigma}$ representing the reduced maps of $S, S^{\prime}$, respectively, such that $H_{\rho} \rightarrow H_{\sigma}$ (delete $I$ ) for some array $I$, of length 2 , whose elements are $a, b . \sigma=\rho$ except on the set $\{a, b\} ; a \sigma=b, b \sigma=a$. Let $H_{\rho} \cong X I Y$ and $H_{\sigma} \cong X Y$.

If $S_{i}=\left(E_{i}, \beta_{i}, \rho_{i}, \theta_{i}\right)$ are the minimal substructures of $S(1 \leqq i \leqq r)$, then there exist arrays $M_{1}, \cdots, M_{r}$ representing the reduced maps of $S_{1}, \cdots, S_{r}$, respectively, such that $1 \rightarrow H_{\rho}$ (insert $M_{1}, \cdots, M_{r}$ ). Suppose $a \in E_{i}, b \in E_{j}$.

Case 1. $(i=j)$ Since $E_{i}$ is closed under $\beta$ and $\sigma, E_{i}$ is the carrier of a substructure $S_{i}^{\prime}$ of $S^{\prime}$. The fact that $S_{i}$ is minimal implies that each nonempty proper subset $A$ (of $E_{i}$ ) is not closed under both $\beta$ and $\rho$; hence $A$ is not closed under both $\beta$ and $\sigma$. Thus $S_{i}^{\prime}$ is a minimal substructure of $S^{\prime}$.

Let $M_{i} \cong P I Q$. Then the possibly empty array $M_{i}^{\prime} \cong P Q$ represents the reduced map of $S_{i}^{\prime}$. Finally, $1 \rightarrow H_{\sigma}$ (insert $M_{1}, \cdots, M_{i-1}, M_{i}^{\prime}, M_{i+1}$, $\left.\cdots, M_{r}\right)$. 
Case 2. ( $i<j)$ By Lemma 6.1, there is an array $K$ such that $1 \rightarrow H_{\rho} \quad$ (insert $\left.M_{1}, \cdots, M_{i-1}, K, M_{i+1}, \cdots, M_{j-1}, M_{j+1}, \cdots, M_{r}\right), \quad 1 \rightarrow K$ (insert $M_{i}, M_{j}$ ), and $K$ is of the form $K \cong P I Q$.

$E_{i}, E_{j}$, and hence $E_{i} \cup E_{j}$ are closed under $\beta$ and $\rho$. Then $E_{i} \cup E_{j}$ is closed under $\sigma$ and is the carrier of a substructure $S_{i}^{\prime}$ of $S^{\prime}$. Since $E_{i}, E_{j}$, and each nonempty proper subset of either $E_{i}$ or $E_{j}$ are not closed under both $\beta$ and $\sigma$, we have that $S_{i}^{\prime}$ is a minimal substructure of $S^{\prime}$.

The possibly empty array $K^{\prime} \cong P Q$ represents the reduced map of $S_{i}^{\prime} ; 1 \rightarrow H_{\sigma}$ (insert $M_{1}, \cdots, M_{i-1}, K^{\prime}, M_{i+1}, \cdots, M_{j-1}, M_{j+1}, \cdots, M_{r}$ ). This completes the proof of Lemma 9.1.

Now Theorem 9.1 can be proved. Let $S=(E, \beta, \rho, \theta)$ be a structure with $k \rho$-cycles of length 2. If $k=0$, then $\rho$ is the identity, the $\beta$-orbits are the carriers of the minimal substructures of $S$, and $\theta$ is the reduced map of $S$. Theorem 9.1 holds in this case since $\theta$ splits into the $\beta$-cycles (by the definition of a structure).

If $k \geqq 1$, then there exist structures $T_{0}=\left(E, \beta, \rho_{0}, \theta\right), \cdots, T_{k}=$ $\left(E, \beta, \rho_{k}, \theta\right)$ where $\rho_{0}$ is the identity and $\rho_{k}=\rho, T_{k}=S$ such that $T_{i}$ contracts to $T_{i+1}$ in one step $(0 \leqq i \leqq k-1)$. Use Lemma 9.1 and the fact that $T_{0}$ satisfies Theorem 9.1 to get that $T_{k}=S$ satisfies Theorem 9.1. This completes the proof.

Since each relator is a value of the reduced map of some $\mathscr{R}$ structure, we have

COROLlaRY 9.1. Each relator splits into minimal relators.

The next 3 lemmas will be useful later.

LEMMA 9.2. A nonminimal relator, of type $n \geqq 2$, splits into relators having types smaller than $n$.

Proof. Observe that a relator of type 1 is necessarily minimal. Use Theorem 9.1 and the fact that a nonminimal structure, of type $n \geqq 2$, has minimal substructures whose types have sum $n$.

LEMMA 9.3. Let $S=(E, \beta, \rho, \theta)$ be a structure. If the array $H \cong a c_{1} \cdots c_{r} b D, \quad r \geqq 1$, represents $\theta$ and if the fixed elements $a, b$ satisfy $a \beta=b$, then $\left\{c_{1}, \cdots, c_{r}\right\}$ is closed under $\beta$ and $\rho$.

Proof. There exist arrays $H_{1}, \cdots, H_{n}$ representing the $\beta$-cycles $\mu_{1}, \cdots, \mu_{n}$, respectively, such that $1 \rightarrow H$ (insert $H_{1}, \cdots, H_{n}$ ). Since $a \beta=b, a b$ is a subword of $H_{i}$ for some $i, 1 \leqq i \leqq n$. Since $a b$ is not a subword of $H$, we have $i<n$. The set $\left\{c_{1}, \cdots, c_{r}\right\}$ must be the union of the $\beta$-orbits corresponding to some subsequence of $\mu_{i+1}, \cdots$, 
$\mu_{n}$. Hence, $\left\{c_{1}, \cdots, c_{r}\right\}$ is closed under $\beta$.

Since $a, b$ are fixed elements, we have that $\left\{c_{1}, \cdots, c_{r}\right\}$ is closed under $\rho$.

LEMma 9.4. Let $a, b$ be fixed elements of a minimal structure $S=(E, \beta, \rho, \theta)$ with reduced map $\theta_{F}$. If $a \beta=b$, then $a \theta=b$ and $a \theta_{F}=b$.

Proof. If $\mathrm{a} \theta \neq b$, then there is an array $a c_{1} \cdots c_{r} b, r \geqq 1$, which partially represents $\theta$. Lemma 9.3 implies that $\left\{c_{1}, \cdots, c_{r}\right\}$ is the carrier of a proper substructure of $S$. This is impossible since $S$ is minimal. Thus, $a \theta=b$. But then $a \theta_{F}=b$ since $a, b$ are fixed elements.

10. Asymmetric relators. Let $W$ be an $\mathscr{R}$-word with $1 \rightarrow W$ (insert $R_{1}, \cdots, R_{n}$ ) where the $R_{i}$ are defining relators. We always consider just one mode of performing the insertions (if there is more than one). Since each letter of $W$ originates from a letter of one of the $R_{i}$, there is a one-to-one correspondence between the letters in $W$ and the letters in $R_{1}, \cdots, R_{n}$.

Let $X \cong X_{1} x X_{2}$ and $Y \cong Y_{2} y Y_{1}$ be any two of the $R_{i}$. Suppose that $x, y$ correspond to the letters $u, v$ in $W$; that $u, v$ can cancel with each other during free reduction of $W$; and that the words $X_{2} X_{1} x$ and $y Y_{1} Y_{2}$ are inverses. Then we say that $u, v$ can cancel symmetrically or that $W$ is a symmetric $\mathscr{R}$-word.

In this situation, either $u, v$ are adjacent in $W$ or $u, v$ are separated by a nonempty subword (of $W$ ) which freely reduces to 1 . We indicate this by saying that $u, v$ can cancel either immediately or eventually; $W$ is either immediately or eventually symmetric. If no two letters of $W$ can cancel symmetrically during free reduction of $W$, then $W$ is an asymmetric $\mathscr{R}$-word. Finally, an asymmetric (symmetric) relator of type $t$ is a partially reduced asymmetric (symmetric) $\mathscr{R}$-word of type $t$.

LEMMA 10.1. If a word $W$ splits into $t \geqq 2$ defining relators, two of which are $X, Y$, then $W$ splits into two words $U, V$ such that $U$ splits into $p \geqq 1$ defining relators, one of which is $X$, $V$ splits into $q \geqq 1$ defining relators, one of which is $Y$, and $p+q=t$.

Proof. Use induction on $t$. The lemma holds for $t=2$ with $U \cong X, V \cong Y$. Let $t \geqq 3$ and assume the lemma is true for smaller $t$. Suppose $1 \rightarrow W$ (insert $R_{1}, \cdots, R_{t}$ ) and $X \cong R_{i}, Y \cong R_{j}$ for $i<j$. Let $W^{\prime}$ be the word such that $1 \rightarrow W^{\prime}$ (insert $R_{1}, \cdots, R_{t-1}$ ) and $W^{\prime} \rightarrow W$ (insert $R_{t}$ ). 
If $j=t$, choose $U \cong W^{\prime}, V \cong R_{t}$. If $j<t$, then by the induction assumption $W^{\prime}$ splits into two words $U^{\prime}, V^{\prime}$ which split into $p^{\prime}$ defining relators and $q^{\prime}$ defining relators among which are $X$ and $Y$, respectively, where $p^{\prime}+q^{\prime}=t-1$. We can choose $U, V$ so that either $U \cong U^{\prime}$ and $V^{\prime} \rightarrow V$ (insert $R_{t}$ ) or $V \cong V^{\prime}$ and $U^{\prime} \rightarrow U$ (insert $R_{t}$ ).

LEMMA 10.2. An eventually symmetric $\mathscr{R}$-word $W$, of type $t \geqq 2$, is freely equal to some immediately symmetric $\mathscr{R}$-word $W^{\prime}$, of type $t$.

Proof. Suppose $1 \rightarrow W$ (insert $R_{1}, \cdots, R_{t}$ ) where the $R_{k}$ are defining relators. Let $W$ contain the letters $u, v$ which can eventually cancel symmetrically during free reduction of $W$. Suppose that $u, v$ correspond to the letters $x, y$ in $R_{i} \cong X_{1} x X_{2}, R_{j} \cong Y_{2} y Y_{1}$. Apply the previous lemma with $X \cong R_{i}, Y \cong R_{j}$ to find the words $U, V$. Then $U, V$ have cyclic permutations $U^{\prime}, V^{\prime}$, respectively, such that the product $U^{\prime}, V^{\prime}$ is a cyclic permutation of $W$.

Let $U^{\prime} \cong M_{1} m M_{2}$ and $V^{\prime} \cong N_{2} \cong N_{2} n N_{1}$ where $m, n$ correspond to $x, y$, respectively. Since $u, v$ can cancel in $W$, either $N_{1} M_{1}$ or $M_{2} N_{2}$ freely reduces to 1 . Thus $W$ has a cyclic permutation $m M_{2} N_{2} n N_{1} M_{1}$ which partially reduces to either $M_{2} N_{2}$ or $N_{1} M_{1}$.

Put $W^{\prime \prime} \cong M_{2} M_{1} m n N_{1} N_{2}$ which is an $\mathscr{R}$-word of type $t$. In fact, $M_{2} M_{1} m$ is a cyclic permutation of $U$ and is an $\mathscr{R}$-word of the same type as $U$ by Remark 2.1. Similarly, $n N_{1} N_{2}$ and $V$ are $\mathscr{R}$-words of the same type. Thus $W^{\prime \prime}$ is a product of $\mathscr{R}$-words whose types have sum $t$.

Either $W^{\prime \prime}$ partially reduces to $M_{2} M_{1}$ or $W^{\prime \prime}$ has a cyclic permutation which partially reduces to $N_{1} M_{1}$. Thus $W^{\prime \prime}$ has a cyclic permutation $W^{\prime}$ which is freely equal to $W$.

LEMMA 10.3. Let $W$ be a word which splits into $t \geqq 2$ defining relators $R_{1}, \cdots, R_{t}$. If two letters $u, v$ in $W$ can immediately cancel symmetrically, then $W$ also splits into $t-2$ defining relators and one or more null words.

Proof. Let $R_{i} \cong X_{1} x X_{2}$ and $R_{j} \cong Y_{2} y Y_{1}$ where $x, y$ correspond to $u, v$, respectively. By assumption, $X_{2} X_{1} x$ and $y Y_{1} Y_{2}$ are inverses so that $X_{1} x y Y_{1} Y_{2} X_{2}$ and $X_{1} Y_{1} Y_{2} y x X_{2}$ freely reduce to 1 .

The proof of Lemma 6.1 shows that $W$ splits into $t-2$ defining relators and a word $U$. Either $U \cong X_{1} x y Y_{1} X_{2}$ (with $Y_{2} \cong 1$ ) or $U \cong$ $X_{1} Y_{2} y x X_{2}$ (with $Y_{1} \cong 1$ ). In either case, $U$ freely reduces to 1 so that $U$ splits into one or more null words. Thus, $W$ splits into $t-2$ defining relators and one or more null words. 
Lemma 10.4. Suppose $1 \rightarrow U$ (insert $X, Y$ ) where $X, Y$ are relators of types $p, q \geqq 0$ with the understanding that a relator of type 0 is a null word. Let $U$ have a subword $N$ which is a null word whose letters $u, v$ correspond to a letter in $X$ and a letter in $Y$, respectively. Let $V$ be defined by $U \rightarrow V$ (delete $N$ ). Then $V$ is a relator of type $p+q$.

Proof. If $p=q=0$, then $X, Y$ and hence $V$ are null words. If $p>0, q=0$, then $V \cong X$. If $p=0, q>0$, then either $V \cong Y$ or $V$ is a cyclic permutation of $Y$.

Finally, if $p>0, q>0$, then $X, Y$ are partially reduced forms of $\mathscr{R}$-words $P, Q$ of types $p, q$, respectively. Then $U$ is a partially reduced form of an $\mathscr{R}$-word $M$, of type $p+q$, such that $1 \rightarrow M$ (insert $P, Q)$. Thus $U$ is a relator of type $p+q$; hence so is $V$.

LEMMA 10.5. If a word $W$ splits into null words and/or relators having types whose sum is $t \geqq 1$, then this is also true for each word $W^{\prime}$ which is freely equal to $W$.

Proof. It suffices to check the cases when $W^{\prime}$ is obtained from $W$ by a single insertion or deletion of a null word $N$. If $W \rightarrow W^{\prime}$ (insert $N$ ), then $W^{\prime}$ satisfies the lemma.

Now suppose $W \rightarrow W^{\prime}$ (delete $N$ ). By assumption $1 \rightarrow W$. (insert $W_{1}, \cdots, W_{r}$ ) where $W_{1}, \cdots, W_{r}$ are null words and/or relators having types whose sum is $t$. Let $W_{k}$ have type $t_{k}$ with $t_{k}=0$ if $W_{k}$ is a null word. The lemma holds when each $W_{k}$ is a null word since then $W^{\prime}$ also splits into null words. Therefore, assume some $W_{k}$ is not a null word so that $t_{1}+\cdots+t_{r}=t$.

One possibility is that the letters in $N$ correspond to letters in the same $W_{i}$ so that $W_{i} \rightarrow W_{i}^{\prime}$ (delete $N$ ) for some word $W_{i}^{\prime}$. If $t_{i}=0$, $W_{i}^{\prime}$ is the empty word. If $t_{i} \geqq 1, W_{i}^{\prime}$ is either empty or a relator of type $t_{i}$. In any case, $1 \rightarrow W^{\prime}$ (insert $W_{1}, \cdots, W_{i-1}, W_{i}^{\prime}, W_{i+1}, \cdots, W_{r}$ ).

The other possibility is that the letters in $N$ correspond to letters in two words $W_{i}, W_{j}$ so that $r \geqq 2$. Lemma 6.1 implies that $W$ splits into $r-2 W_{k}^{\prime}$ 's, having types whose sum is $t-t_{i}-t_{j}$, and a word $U$ which splits into $W_{i}, W_{j}$. Then $W^{\prime}$ splits into the same $r-2 W_{k}$ 's and a word $V$ such that $U \rightarrow V$ (delete $N$ ). By the previous lemma, $V$ is a relator of type $t_{i}+t_{j}$. This completes the proof.

LemMa 10.6. A symmetric relator $W$, of type $t \geqq 2$, splits into null words and/or relators having types smaller than $t$. 
Proof. Let $W$ be a partially reduced form of a symmetric $\mathscr{R}$-word $V$ of type $t$. By Lemma $10.2 V$ is freely equal to an immediately symmetric $\mathscr{R}$-word $V^{\prime}$ of type $t$. By Lemma 10.3 either $t=2$ and $V^{\prime}$ splits into null words or $t \geqq 3$ and $V^{\prime}$ splits into null words and relators having types whose sum is $t-2$, (since a defining relator is a relator of type 1). By Lemma 10.5, $W$ splits into null words and/or relators having types whose sum is $t-2$. This implies Lemma 10.6.

THEOREM 10.1. Each relator splits into null words and/or asymmetric relators.

Proof. Let $W$ be a relator of type $t \geqq 1$. When $t=1, W$ is a defining relator which is an asymmetric relator. Use induction on $t$. Let $t \geqq 2$ and assume the theorem for relators of type smaller than $t$. Theorem 10.1 then follows from Lemma 10.6.

11. Proof of Main Theorem. In order to solve the word problem in the presented group $\mathscr{G}$, it suffices to be able to recognize the asymmetric, minimal relators which we call basic relators.

THEOREM 11.1. Each relator splits into null words and/or basic relators.

Proof. Use Lemma 9.2, Lemma 10.6 and the fact that a relator of type 1 (a defining relator) is a basic relator. This completes proof.

We now consider a basic relator in a sixth group. More specifically, consider a cyclically reduced relator $W$ which is a value of the reduced map of a minimal, noncancelled $\mathscr{R}$-structure $S=(E, \beta, \rho, \theta)$, of type $n \geqq 2$. Then some cyclic permutation of $W$ is the freely reduced form of an $\mathscr{R}$-word $V$ of type $n$, where $V$ is a value of $\theta$. We assume that $V$ is an asymmetric $\mathscr{R}$-ward so that $W$ is an asymmetric relator. The structure $S$ characterizes one method of freely reducing $V$ to a word which is a cyclic permutation of $W$. As usual, let $S_{1}=\left(E_{1}, \beta_{1}, \rho_{1}, \theta_{1}\right)$ be the cancelled $\mathscr{N}$-structure associated with $S ; C_{1}=\left(E_{1}, \beta_{1}, \rho_{1}\right)$. Note that $C_{1}$ has no vertex containing just one edge (by Theorem 7.1).

In this situation, consider the $B_{k}^{i}$ of $\S 8$. The following lemma implies that $B_{1}^{1}=B_{2}^{2}=B_{3}^{2}=0$.

LEMma 11.1. Let $S=(E, \beta, \rho, \theta)$ be a noncancelled, minimal structure with associated cancelled structure $S_{1}=\left(E_{1}, \beta_{1}, \rho_{1}, \theta_{1}\right)$. Let $C_{1}=\left(E_{1}, \beta_{1}, \rho_{1}\right)$ and assume that $C_{1}$ has no vertex containing just one edge. Suppose the product $X Y$ of nonempty arrays partially represents a nondistinguished $\beta_{1}$-cycle and $X, Y$ are both sides in $C_{1}$. 
Then $X, Y$ are not both fixed sides. Also, there is no nondistinguished $\beta_{1}-c y c l e$ which is represented by one fixed side.

Proof. Suppose $X, Y$ are fixed sides. This assumption together with the fact that $X Y$ partially represents a nondistinguished $\beta_{1}$-cycle imply that $X Y$ partially represents $\theta_{F}$, the reduced map of $S$. Let $a$ be the last letter in $X$; let $b$ be the first letter in $Y$. Since $Y$ is a side of $C_{1}, b$ is an initial edge. Also, since $\{b\}$ cannot be a vertex of $C_{1}$, we have $b \rho_{1} \beta_{1} \neq b$.

Since $a, b$ are fixed elements of $S$ and $a \beta=a \beta_{1}=b$, we have $a \theta_{F}=b$ by Lemma 9.4. By Remark $5.1 b \rho_{1} \beta_{1}=a \rho_{1}$. Hence $b \rho_{1} \beta_{1} \rho_{1} \beta_{1}=$ $a \rho_{1} \rho_{1} \beta_{1}=a \beta_{1}=b$. This contradicts the fact that $b$ is an initial edge of $C_{1}$. Thus, both $X$ and $Y$ cannot be fixed sides.

Now let $Z$ be a fixed side, representing a nondistinguished $\beta_{1}$-cycle. If $Z$ is of length $\geqq 2$, let $a, b$ be the last and first letters of $Z$, respectively, so that $a \neq b$. We get a contradiction as before.

If $Z$ is of length 1 and $Z \cong a$, then $a \beta=a \beta_{1}=a$ and $a \rho=a$. Hence, $\{a\}$ is the carrier of a proper substructure of $S$, which is again a contradiction. This completes the proof.

Let the arrays $M X$ and $Y N$ represent nondistinguished $\beta_{1}$-cycles $\mu, \nu$. respectively. Assume that the values of $M X, Y N$ are the defining relators $R_{1}, R_{2}$, respectively, and that $X, Y$ are inverse sides.

If $\mu \neq \nu$, then $R_{1}, R_{2}$ are not inverses since $V$ is asymmetric. Hence, $R_{1}$ and $R_{2}^{-1}$ are distinct defining relators with a common subword (the value of $X$ ). The less-than-one-sixth property implies that

$$
\text { (*) } \quad l(X)<\frac{1}{6} l(M X) \text { and } l(Y)<\frac{1}{6} l(Y N) .
$$

It is also possible that $\mu=\nu$. In this case $R_{1}, R_{2}$ are cyclic permutations of one another. Once again (*) will hold provided that $R_{1}, R_{2}$ are not inverses. But this proviso holds.

LEMMA 11.2. If $T$ is a nonempty cyclically reduced word, then no cyclic permutation of $T$ is the word $T^{-1}$.

Proof. Let $U \cong T_{2} T_{1}$ be a cyclic permutation of $T \cong T_{1} T_{2}$. If $U \cong T^{-1}$, then $T_{1} \cong T_{1}^{-1}, T_{2} \cong T_{2}^{-1}$; hence $T_{1}, T_{2}$ are empty words, contradiction.

Thus, for $C_{1}$, we also have $B_{k}^{0}=0$ for $1 \leqq k \leqq 6$. From (6) in $\S 8$, we get $3 B_{2}^{1}+2 B_{3}^{1}+B_{4}^{1} \geqq 6$. This implies the Main Theorem with $P_{k}=B_{k}^{1}, k=2,3,4$. 


\section{REFERENCES}

1. R. Courant, and H. Robbins, What is Mathematics?, New York, Oxford University Press, 1941.

2. M. Greendlinger, Dehn's algorithm for the word problem, Comm. Pure Appl. Math. 13 (1960), 67-83.

3. V. A. Tartakovskii, Amer. Math. Soc. Translation No. 60. Translation of Three Russian Papers: The sieve method in group theory, Mat. Sbornik N. S. 25 (67), (1949), 3-50. Application of the sieve method to the word problem for certain types of groups, Mat. Sbornik N. S. 25 (67), (1949), 251-274. Solution of the word problem for groups. with a k-reduced basis $k>6$, Izvestiya Akad. Nauk SSSR Ser. Mat. 13 (1949), 483-494. 4. E. R. Van Kampen, On some lemmas in the theory of groups, Amer. J. Math. 55 (1933), 268-273.

UNIVERSITY OF CALIFORNIA, LOS ANGELES 


\section{PACIFIC JOURNAL OF MATHEMATICS}

\section{EDITORS}

\author{
H. SAMmLSON \\ Stanford University \\ Stanford, California \\ R. M. BLUMENTHAL \\ University of Washington \\ Seattle, Washington 98105
}

\author{
*J. DUGUNDJI \\ University of Southern California \\ Los Angeles, California 90007
}

RICHARD ARENS

University of California

Los Angeles, California 90024

\section{E. F. BECKENBACH \\ B. H. NEUMANN \\ ASSOCIATE EDITORS}

\section{SUPPORTING INSTITUTIONS}

\author{
UNIVERSITY OF BRITISH COLUMBIA \\ CALIFORNIA INSTITUTE OF TECHNOLOGY \\ UNIVERSITY OF CALIFORNIA \\ MONTANA STATE UNIVERSITY \\ UNIVERSITY OF NEVADA \\ NEW MEXICO STATE UNIVERSITY \\ OREGON STATE UNIVERSITY \\ UNIVERSITY OF OREGON \\ OSAKA UNIVERSITY \\ UNIVERSITY OF SOUTHERN CALIFORNIA
}

\author{
STANFORD UNIVERSITY \\ UNIVERSITY OF TOKYO \\ UNIVERSITY OF UTAH \\ WASHINGTON STATE UNIVERSITY \\ UNIVERSITY OF WASHINGTON \\ * * * \\ AMERICAN MATHEMATICAL SOCIETY \\ CHEVRON RESEARCH CORPORATION \\ TRW SYSTEMS \\ NAVAL ORDNANCE TEST STATION
}

\footnotetext{
Mathematical papers intended for publication in the Pacific Journal of Mathematics should be typewritten (double spaced). The first paragraph or two must be capable of being used separately as a synopsis of the entire paper. It should not contain references to the bibliography. Manu. scripts may be sent to any one of the four editors. All other communications to the editors should be addressed to the managing editor, Richard Arens at the University of California, Los Angeles, California 90024.

50 reprints per author of each article are furnished free of charge; additional copies may be obtained at cost in multiples of 50 .
}

The Pacific Journal of Mathematics is published monthly. Effective with Volume 16 the price per volume (3 numbers) is $\$ 8.00$; single issues, $\$ 3.00$. Special price for current issues to individual faculty members of supporting institutions and to individual members of the American Mathematical Society: $\$ 4.00$ per volume; single issues $\$ 1.50$. Back numbers are available.

Subscriptions, orders for back numbers, and changes of address should be sent to Pacific Journal of Mathematics, 103 Highland Boulevard, Berkeley 8, California.

Printed at Kokusai Bunken Insatsusha (International Academic Printing Co., Ltd.), No. 6, 2-chome, Fujimi-cho, Chiyoda-ku, Tokyo, Japan.

PUBLISHED BY PACIFIC JOURNAL OF MATHEMATICS, A NON-PROFIT CORPORATION

The Supporting Institutions listed above contribute to the cost of publication of this Journal, but they are not owners or publishers and have no responsibility for its content or policies.

* Paul A. White, Acting Editor until J. Dugundji returns. 


\section{Pacific Journal of Mathematics}

\section{Vol. 16, No. $3 \quad$ BadMonth, 1966}

Gert Einar Torsten Almkvist, Stability of linear differential equations with

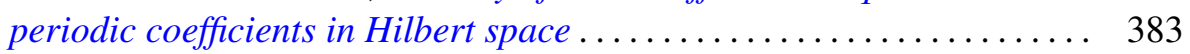

Richard Allen Askey and Stephen Wainger, A transplantation theorem for ultraspherical coefficients ................................ 393

Joseph Barback, Two notes on regressive isols .................. 407

Allen Richard Bernstein and Abraham Robinson, Solution of an invariant subspace problem of K. T. Smith and P. R. Halmos .............. 421

P. R. Halmos, Invariant subspaces of polynomially compact operators . . . . 433

Leon Bernstein, New infinite classes of periodic Jacobi-Perron algorithms.................................... 439

Richard Anthony Brualdi, Permanent of the direct product of matrices .... . 471

W. Wistar (William) Comfort and Kenneth Allen Ross, Pseudocompactness and uniform continuity in topological groups .................. 483

James Michael Gardner Fell, Algebras and fiber bundles . . . . . . . . . . . . 497

Alessandro Figà-Talamanca and Daniel Rider, A theorem of Littlewood and lacunary series for compact groups ..................... 505

David London, Two inequalities in nonnegative symmetric matrices...... 515

Norman Jay Pullman, Infinite products of substochastic matrices ........ 537

James McLean Sloss, Reflection and approximation by interpolation along

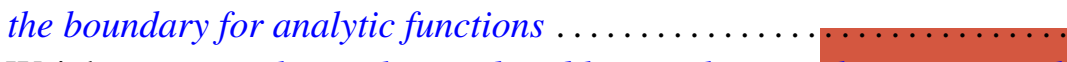

Carl Weinbaum, Visualizing the word problem, with an application to sixth groups................................... 